\title{
天敌伴迁与生物多样性
}

翟保平

(南京农业大学昆虫学系, 农业部病虫监测与治理重点实验室，南京 210095)

摘要 : 在对昆虫迁飞进行雷达观测时发现, 天敌 (包括蜘蛛) 种群的大规模伴迁现象引人注目, 国内外的空捕、海捕 中也都有天敌入网。这种同步迁移现象无疑具有重要的生态学和行为学意义, 表现出 天敌一寄主一植物”不同营 养层之间的相互作用。而温带和热带空中取样中伴迁天敌数量相差之巨, 也突出反映了不同的生物多样性背景。 对低层大气中的生物流量进行长期监测, 可望从另一个层面提供可靠的生物多样性信息。

关键词: 天敌, 伴迁, 生物多样性

中图分类号 :S476,S763.306.4 文献标识码 : A 文章编号 : 1005-0094(2001)02-0176-05

\section{Accompanying migration of natural enemies and biodiversity}

ZHAI Bao-Ping

Key Laboratory of Pest Monitoring and Management of Agricultural Ministry of China ,Department of Entomology, Nanjing Agricultural University, Nanjing 210095

\begin{abstract}
It was found in radar observations and marine samples that the phenomena of accompanying migration by natural enemies (including aeronautic spiders) with their hosts was significant and spectacular. The phenomena shows that the interaction between different trophic levels of natural enemy-insect and pest-plant is undoubtedly of important ecological and behavioral significance. The great difference of natural enemy catches in aerial samples between different areas in the tropics and the temperate zone reflects the different backgrounds of biodiversity. The long-term monitoring of bio-flux in the low-level atmosphere would provide reliable information on biodiversity.
\end{abstract}

Key words : bio-flux , migration, natural enemy

近年来,国内外的雷达观测中屡屡发现天敌的 大规模伴迁现象,在不同地区的空捕和海捕中也都 有天敌入网 (Mochida \& Takada, 1978;Greenstone et al. ,1987 ; Reynolds \& Wilson ,1989 ; Riley et al. , 栙 世良等,1986; 朱明华,1989;罗瑞梧等,1994; 吴孔 明郭予元，1998）,这种天敌与害虫同步迁移现象 无疑具有重要的生态学和行为学意义。迁移是生物 对栖息环境和资源配置的季节性变化所表现出的适 应性行为,害虫的远距离迁飞往往导致其在农林牧 区突发成灾, 对农林牧业生产造成严重威胁。我国 地处东亚季风区, 特殊的地理气候条件使我国农作 物的主要害虫都是南北往返迁飞数千公里, 往往 小虫成大灾”。人们已经注意到, 近年来迁飞性害 虫猖獗频率的不断增加正是因滥用农药、破坏生物
多样性所致 ( Heinrichs \& Mochida ,1984;Hardin et al. 1995;Way \& Heong, 1994) , 因而应提倡维护非 农田生境以保持害虫天敌稳定的栖息环境并提供避 难所 (俞晓平等,1998)。本文对天敌伴迁现象做一 综述, 阐释这一现象对生物多样性保持和害虫综合 治理的意义。

\section{1 捕食性天敌的伴迁}

水稻在我国的粮食生产中具有举足轻重的地 位。在粮食作物中, 水稻以占总面积 $26 \%$ 的耕地， 产出占总产量 $43 \%$ 的粮食, 对我国粮食的增产贡献 最大。同时, 我国农作物的重大害虫中, 水稻害虫占 半数之多且多具迁飞性, 其中褐飞闽( Nilaparvata lugens) 和白背飞虫 ( Sogatella furcifera) 20 世纪 80 年 
代以来就有十几年大发生, 尤以 1987 年和 1991 年 两次暴发成灾时损失最为惨重。

对迁飞性害虫而言，迁入区的天敌种群因跟随 效应而不大容易起到控害作用，但伴迁的天敌则在 一定情况下有可能发挥其抑制功能。梁佳学等 (1991) 发现若干褐飞闽 ( Nolaparvate lugens) 迁入峰 后并未出现相应的若虫峰, 分析其原因可能是伴迁 天敌的抑制作用所致 (翟保平,1992)。黑肩绿盲蝽 (Cyrtorhinus lividipennis) 和小宽蝽 (Microvelia atrolineata ) 是捕食褐飞風、白背飞虫和叶蝉等害虫卵和 若虫的重要天敌。朱明华 (1989) 的研究表明,在迁 入早、迁入量大的年份 (如 1987 年),黑肩绿盲蝽对 稻田内飞虫和叶蝉的控制作用非常明显。Cook \& Perfect (1985) 也曾报道过，黑肩绿盲蝽和小宽蝽的 迁入率与褐飞風和白背飞闽的种群发展呈显著负相 关, 显示出伴迁天敌对害虫种群的调控作用。曾有 关于黑肩绿盲蝽与褐飞虫和白背飞虫同步起飞的报 道（朱明华，1989;Ooi，1979），国内外的空捕（邓望 喜, 1981; 广东省农科院植保所迁飞性害虫研究组, 1979 )、海捕(刘浩官等, 1983; 岸本良一, 1975;Kisimoto ,1991) 、空中取样 (Reynolds \& Wilson ,1989;Riley et al. 1987,1991,1994)、高山网(全国褐稻虫科 研协作组，1981）等都有黑肩绿盲蝽入网的记录。 而且雷达观测时的空中取样发现, 入网昆虫中除了
大量的褐飞風、白背飞虫以及其他飞虫类、叶蝉类等 害虫外, 黑肩绿盲蝽也占很大比例, 在热带地区要比 飞闽数量大一个量级 (Riley et al. ,1987)。在印度 的空中取样也得到了相似的结果 (Reynolds \& Wilson, 1989;Riley et al. ,1995) ,这与温带地区的情况 截然相反( Riley et al. 1991,1994)(表 1)。

菲律宾群岛属热带海洋性气候, 植物种类丰富， 多样性指数高, 星罗棋布的非稻田生境为天敌昆虫 提供了良好的避难所，因此空中天敌密度是稻飞虫 的 15 倍; 印度次大陆盛行热带季风，其稻区主要分 布于科罗曼德尔沿岸的三角洲, 靠运河和井水灌溉 植稻, 生物多样性丰富程度次于东南亚, 空中天敌种 群的密度是稻飞虫的 8 倍 我国东部盛行温带季风， 江淮稻区正是我国人口最密集之地, 人均几分地上 的精耕细作, 就连田埂上的杂草也被铲光 (值得指 出的是, 我国的综防措施中往往包括铲除地边杂草 一项）,因此几乎无非稻田生境可言,导致天敌数量 急剧减少。尤其在大发生和重发生年( 如 1988 年), 天敌的密度不足稻飞風密度的 $2 \%$ 。如此巨大 的反差, 生动地反映了生物多样性降低与害虫猖獗 的内在联系; 而当害虫因寄主植物分布的季节性变 化迁出原栖息地后，天敌随之而来的跟踪追击则真 实地揭示了“天敌一寄主一植物”不同营养层之间 的相互作用。

表 1 热带和温带稻区空中群落中的稻飞闽及其天敌的数量

Table 1 The aerial net catches of rice planthoppers and its natural enemies in the tropics and temperate areas

\begin{tabular}{|c|c|c|c|c|c|c|}
\hline $\begin{array}{c}\text { 地点 } \\
\text { Location }\end{array}$ & $\begin{array}{l}\text { 时间 } \\
\text { Time }\end{array}$ & $\begin{array}{c}\text { 种类 } \\
\text { Species }\end{array}$ & $\begin{array}{l}\text { 数量 } \\
\text { No. }\end{array}$ & $\begin{array}{l}\text { 占总量比例 } \\
\text { (\%) Percentage }\end{array}$ & $\begin{array}{c}\text { 密度 }\left(\text { 个 } / 10^{4} \mathrm{~m}^{3}\right) \\
\text { Aerial density } \\
\left(\operatorname{nos} . / 10^{4} \mathrm{~m}^{3}\right)\end{array}$ & $\begin{array}{c}\text { 文献 } \\
\text { Reference }\end{array}$ \\
\hline 菲律宾 & 1987 & Nilaparvata lugens & 148 & 5.9 & 4.16 & \multirow{4}{*}{$\begin{array}{l}\text { Riley et al. , } \\
1987\end{array}$} \\
\hline Hoechst , Philippines & & Sogatella furcifera & 29 & 1.2 & - & \\
\hline $14^{\circ} 10^{\prime} \mathrm{N}$ & & Cyrtorhinus lividipennis & 1405 & 56.3 & 60.91 & \\
\hline $121^{\circ} 18^{\prime} \mathrm{E}$ & & Microvelia atrolineata & 53 & 2.1 & 1.81 & \\
\hline 印度中部 & 1985 & Nilaparvata lugens & 52 & 4.1 & 0.60 & \multirow{5}{*}{$\begin{array}{l}\text { Reynolds and } \\
\text { Wilson , } 1989\end{array}$} \\
\hline ICRISAT , & & Sogatella furcifera & 12 & 1.0 & 0.10 & \\
\hline Hyderabad, India & & Cyrtorhinus lividipennis & 339 & 27.0 & 4.60 & \\
\hline $17^{\circ} 28^{\prime} \mathrm{N}$ & & Microvelia atrolineata & 121 & 9.6 & 2.30 & \\
\hline $78^{\circ} 42^{\prime} \mathrm{E}$ & & & & & & \\
\hline 中国江浦 & 1988 & Nilaparvata lugens & 5153 & 57.5 & $8.7 \sim 274.2$ & \multirow{4}{*}{$\begin{array}{l}\text { Riley et al. , } \\
1991\end{array}$} \\
\hline Jiangpu, China & & Sogatella furcifera & 104 & 1.2 & - & \\
\hline $32^{\circ} 03^{\prime} \mathrm{N}$ & & Cyrtorhinus lividipennis & 74 & 0.8 & - & \\
\hline $118^{\circ} 37^{\prime} \mathrm{E}$ & & & & & & \\
\hline 中国江浦 & 1990 & Nilaparvata lugens & 469 & 23.1 & $0.55 \sim 13.34$ & \multirow{3}{*}{$\begin{array}{l}\text { Riley et al. , } \\
1994\end{array}$} \\
\hline Jiangpu , & & Sogatella furcifera & 106 & 5.2 & - & \\
\hline China & & Cyrtorhinus lividipennis & 50 & 2.5 & - & \\
\hline
\end{tabular}


表 2 美国密苏里和澳大利亚新南威尔士的空中蜘蛛群落 (Greenstone et al. 1987)

Table 2 The aeronaut faunas over Missouri , USA and New South Wales, Australia(Greenstone et al. , 1987)

\begin{tabular}{|c|c|c|c|c|}
\hline \multirow{2}{*}{$\begin{array}{c}\text { 蜘蛛类群 } \\
\text { Aeronaut faunas }\end{array}$} & \multicolumn{2}{|c|}{ 密苏里 Missouri, USA } & \multicolumn{2}{|c|}{ 新南威尔士 New South Wales, Australia } \\
\hline & 数量 No. & 比例 $(\%)$ Percentage & 数量 No. & 比例 (\%) Percentage \\
\hline 皿蛛科 Linyphiidae & 1037 & 44.9 & 403 & 54.2 \\
\hline 猫蛛科 Oxyopidae & 231 & 10.0 & 139 & 18.7 \\
\hline 蟹蛛科 Thomisidae & 315 & 13.6 & 3 & 0.4 \\
\hline 肖蛸科 Tetragnathidae & 312 & 13.5 & 1 & 0.1 \\
\hline 跳蛛科 Salticidae & 37 & 1.6 & 13 & 1.8 \\
\hline 逍遥蛛科 Philodromidae & 28 & 1.2 & 6 & 0.8 \\
\hline 管巢蛛科 Clubionidae & 8 & 0.4 & 3 & 0.4 \\
\hline
\end{tabular}

除了黑肩绿盲蝽和小宽蝽外，伴迁的捕食性天 敌还报道有微小花蝽 (Orius minutus)、尖钩宽蝽 (Microvelia horvath)、大草蛉 (Chrysopa septempucta$t a)$ 、中华草蛉 (Chrysopa sinica)、龟纹瓢虫( Propylaea japonica)、异色㼼虫 ( Harmonia axyridis)、七星 瓢虫 (Coccinella septempunctata) (Rothschild,1971； Rubin,1981)、针斑长足㼼虫 (Hippodamia convergens) (Rankin \& Rankin ,1980) 和隐翅虫 ( Staphylinidae ) 等。其中异色漂虫和七星瓢虫的迁飞往往与其 越冬越夏的滞育性迁飞有关(罗希成,1964; 蔡晓明 等,1980; 阎浚杰等, 1981; 杨金宽,1984;翟保平 $1990)$ 。在伴迁的捕食性天敌中，飞航的蜘蛛是规 模最大、种类最多的类群。

与昆虫不同, 蜘蛛不具翅, 故只能靠连续吐丝而 被气流运载, 即飞航 ( ballooning) , 以此进行远距离 扩散可达数千公里 (Holzapfel \& Perkins ,1969; Salmon \& Horner , 1977 ; Okuma \& Kisimoto , 1981)。 Greenstone et al. (1987) 报道了在美国密苏里和澳 大利亚新南威尔士进行的飞航蜘蛛采集试验（表 2 ）结果表明，在所得的 2800 多头蜘蛛中，皿蛛科占 半数以上。绝大多数捕获的蜘蛛的体重为 $0.2 \sim 1$. $0 \mathrm{mg}$,最重的个体分别为 $25.5 \mathrm{mg}$ (密苏里) 和 19.1 $\mathrm{mg}$ (新南威尔士)。两地飞航者中分别有 $14 \%$ 和 $32 \%$ 的个体是成蛛，而皿蛛科飞航者中成蛛的比例 在两地分别占 $31 \%$ 和 $58 \%$ 。早期关于皿蛛科飞航 者的报道中也注意到了其成蛛比例高的现象 (Salmon \& Horner ,1977; Meijer,1977) ,这意味着迁入的 成蛛将有较高的生殖价，因而有较大的定殖成功的 可能性。

\section{2 寄生性天敌的伴迁}

国内外的空捕、海捕都有寄生蜂入网的记录。
Mochida \& Takada(1978) 在东海距九州海岸 150 $200 \mathrm{~km}$ 处捕得 105 头从中国大陆越海迁往日本的 蚜茧蜂, 其中, 木通长管蚜茧蜂 (Aphidius picipes) 、烟 蚜茧蜂 (Aphidius gifuensis) 和菜少脉蚜茧蜂 (Diaeretiella rapae) 分别占 $75.2 \% 、 13.3 \%$ 和 $9.5 \%$, 而且所 有入网的蚜茧蜂均为雌蜂。虽然这些蚜茧蜂雌蜂的 成虫寿命较雄蜂稍长 (Aphidius picipes 雌 $9 \sim 15$ 天, 雄 $8 \sim 11$ 天; A. gifuensis 雌 17.6 天, 雄 13.0 天; $D i$ aeretiella rapae 雌 14.7 天，雄 10.7 天）,却并不足以 解释无雄蜂入网的原因。估计可能是两性之间存在 某种影响其跨海远迁能力的飞行行为差异, 也可能 是天敌 - 寄主相互作用中的某种适应与选择。

罗瑞梧等 (1994) 通过 17 年的田间系统调查和 海上网捕，阐明了燕麦蚜茧蜂 (Aphidius avenae) 随麦 长管蚜 (Macrosiphum avenae) 从长江中下游冬麦区 经黄淮海冬麦区到东北春麦区的远距离伴迁, 并分 为早发早控型、早发失控型、晚发失控型和晚发晚控 型四种控蚜类型。他们还发现, 当地羽化及迁出蜂 群的性比接近 1: 1, 而迁飞途中的空中蜂群和迁入 蜂群的雌蜂率均在 $80 \%$ 以上。如山顶上雌蜂率 (外 地或地面迁入) 为 $86.7 \% \sim 100 \%$, 距岸 $312.5 \mathrm{~km}$ 的海岛杂草上为 $81.8 \%, 340$ 头海上入网者的雌蜂 率为 $90.6 \%$ 。这种现象和 Mochida \& Takada(1978) 的报道一致。

除了天敌成虫自身的伴迁，一些天敌种类还以 不同的发育虫态寄生在害虫体内随其远距离迁移。 全国高山网的资料表明，入网者中有被螯蜂、茧蜂、 线虫和跗蛡 (Strepsiptera：Elenchidae) 寄生的个体。 如贵州独山网 1980 年 6 月下旬到 8 月下旬入网的 稻飞闽中有 $10 \% \sim 46.7 \%$ 的个体被跗蛡寄生, 有时 被寄生率可高达 63\% 100\%（全国褐稻禹科研协 
作组，1981）。由此可见，当被寄生率较高或伴迁的 天敌种群数量很大时, 害虫迁入种群的发展将被有 效地抑制。

\section{3 结语}

天敌 (包括蜘蛛) 种群的大规模伴迁现象表现 出 天敌一寄主一植物” 不同营养层之间的相互作 用, 具有重要的生态学和行为学意义;而温带和热带 空中伴迁天敌数量的巨大差别, 突出反映了不同的 生物多样性背景。对低层大气中的生物流量进行长 期监测，可望从另一个层面提供可靠的生物多样性 信息。

生物多样性的测度现在仍有一定的难度, 如物 种丰富度的测度往往受样本大小的影响太大而无法 对不同地区、不同样本的数据进行比较。若使用同 样的测量工具和方法的话, 空中生物流量则是一个 可比的参数。以前的空中生物流量的测定都是用悬 系载网氦气球 (Kytoon) 或载网风筝,但都受风力的 影响而取样高度有限, 且费时费力, 不可能作为长期 监测的工具。ZLC 制式昆虫雷达 (VLR) 的出现使得 空中生物流量的长期监测可望实现 (翟保平 $2001)$ 。这种雷达由电脑控制，全自动运行，雷达信 号可即时分析与处理, 而且有效监测高度可达 2 $\mathrm{km}$,基本上覆盖了整个低层大气，因而是一种理想 的工具。如果能建立一个 VLR 网, 则可得到大范围 的生物流量动态的时间序列，那将使我们对生物多 样性的多尺度时空变化一目了然。美国农业部雷达 组用 VLR 进行了长达两年的连续观测，在昆虫迁飞 行为研究方面取得了卓有成效的进展 (Beerwinkle et al. 1995)。美国的大气生物学联盟 (Alliance for Aerobiology Research，AFAR) 与美国国家气象局的 NEXRAD 多普勒天气雷达网合作, 正在筹建 VLR 网 以实现对全美低层大气 (2 km 以下) 中多种生物的 生物流量进行长期监测( 翟保平,1999)。

另一种有效的方法是英国洛桑试验站利用标准 诱虫灯 (地面) 结合标准吸虫器 (12 $\mathrm{m}$ 高) 的长期联 网监测 (Woiwod \& Harrington,1994)。洛桑试验站 1964 年建立起全英蚜虫监测网，不久又联合欧洲各 国建立了全欧蚜虫监测网。建网的初衷原本是为蚜 虫的预测预报提供基础数据，但建网几十年后的今 天，重新审视所得的历史资料，却得到了完全出乎意 料的收获:几十年生物多样性的时空变化跃然显现
Luff \& Woiwod,1994)。反观我国的情况则令人遗 憾。我国 1973 年开始建成了世界上最大的、独一无 二的病虫监测网和一套完整、规范的监测方法, 可惜 我们缺乏长远的目光面对自身的栖息环境，我们关 心的只是少数几种监测对象 (害虫), 而将其他上灯 的种类 (包括天敌) 全部抛掉, 所以除了虫情, 其他 信息丧失殆尽，因而无法象英国人那样去分析环境 和生物多样性的变化。

但我们眼下至少可以开展以下的工作:在监测 对象中增加若干天敌种类; 在预测中不仅要考虑虫 情和气象, 还要考虑天敌参数及其控害作用; 在防治 决策时应首先考虑使用对天敌杀伤力小的药剂和施 用方法。通过长期的基础数据积累, 才有希望在理 论上真正有所建树，从而在实践上达到质的飞跃。

\section{参考文献}

蔡晓明, 尚玉昌, 阎浚杰, 1980. 中国七星慓虫 (Coccinella septempunctata L.) 迁飞初探. 中国农业科学, 13(1)：74～ 79

邓望喜, 1981。褐飞風及白背飞闽空中迁飞规律的研究. 植 物保护学报, $8(2): 73 \sim 81$

广东省农科院植保所迁飞性害虫研究组, 1979. 褐飞虫迁飞 规律的研究. 广东农业科学, (3)：43 48

李世良, 张凤海, 梁家荣, 刘树法, 1986. 空中昆虫的航捕观 察. 昆虫知识, 23(2): 53 56

梁佳学, 巫国瑞, 俞晓平, 1991. 褐飞風的再迁飞. 病虫测报, 11(4): $59 \sim 60$

刘浩官, 刘振杰, 祝为华, 1983. 我国海上网捕褐稻闽的结 果. 昆虫学报, 26(1)：109 113

罗瑞梧, 杨崇良, 尚佑芬, 李长松, 赵玖华, 1994. 燕麦蚜茧蜂 种群动态及其控蚜效能研究. 植物保护学报, 21(2): $163 \sim 168$

罗希成, 1964. 异色㼼虫越冬集群的报道. 昆虫知识, 8(6): $254 \sim 256$

全国褐稻禹科研协作组, 1981. 高山捕虫网在研究褐飞虫迁 飞规律和预测中的作用。昆虫知识, 18(3)：241 244

吴孔明, 郭予元, 1998. 一代棉铃虫成虫在渤海海面的迁飞 考察. 植物保护学报, 25(4): 337 340

阎浚杰, 尚玉昌, 蔡晓明, 1981. 燕山主峰七星㼼虫迁飞现象 观察. 昆虫知识, 18(5): 214 215

杨金宽, 1984. 长白山异色㼼虫奇斑变㼼虫迁飞时间和种群 成分调查. 森林生态系统研究, 4: 181 184

俞晓平, 胡萃, Heong K L, 1998. 不同生境源的稻飞虫卵寄生 蜂对寄主的选择和寄生特性. 昆虫学报, 41(1)：41４7 翟保平, 1990. 越冬代七星漂虫和异色慓虫的飞翔能力. 应 用生态学报, $\mathbf{1}(3): 214 \sim 220$

翟保平, 1992. 也谈褐飞虫的再迁飞问题。病虫测报, 12 (3) : $36 \sim 40$

翟保平, 1999. 追踪天使一一雷达昆虫学 30 年. 昆虫学报, $\mathbf{4 2}(3): 315 \sim 326$ 
翟保平, 2001. 昆虫雷达: 从研究型到实用型. 遥感学报, 5 (2) : $81 \sim 90$

朱明华. 黑肩绿盲蝽的迁飞观察, 1989. 昆虫知识, 26 (6): $350 \sim 353$

岸本良一, 1975. ウンカ海を渡る. 东京: 中央公论社. 233 pp.

Beerwinkle K R, Lopez J D, Schleider P G and Lingren P D, 1995. Annual patterns of aerial insect densities at altitudes from 500 to 2400 meters in east-central Texas indicated by continuously-operating vertically-oriented radar. Southwestern Entomologist, 18 (Supplement) : $63 \sim 79$

Cook A G and Perfect T J, 1985. The influence of immigration on population development of Nilaparvata lugens and Sogatella furcifera and its interaction with immigration by predators. Crop Protection, 4(4): $423 \sim 433$

Greenstone M H, Morgan C E, Hultsch A L, Farrow R A and Dowse J E, 1987. Ballooning spiders in Missouri, USA, and New South Wales, Australia: family and mass distributions. Journal of Arachnology, 15: $163 \sim 170$

Hardin M R, Benrey B and Coll M, 1995. Arthropod pest resurgence: an overview of potential mechanisms. Crop Protection, 14(1): $3 \sim 18$

Heinrichs E A and Mochida O, 1984. From secondary to major pest status: the case of insecticide-induced rice brown planthopper, Nilaparvata lugens, resurgence. Protection Ecology, 7: $201 \sim 218$

Holzapfel E P and Perkins B D, 1969. Trapping of air-borne insects on ships in the Pacific. Part 7. Pacific Insects, 11: $455 \sim 476$

Kisimoto R, 1991. Long-distance migration of rice insects. In: Heinrichs E A, Miller T A (eds.), Rice Insects: Management Strategies. New York: Springer-Verlag, $167 \sim 195$

Luff M and Woiwod L P, 1994. Insects as indicators of land use change: a European perspective, focusing on moths and ground beetles. In: Harrington R, Stork N E (eds.), Insects in a Changing Environment: Proceedings of the 17th International Symposium of the Royal Entomological Society. London: Academic Press, $214 \sim 236$

Meijer J, 1977. The immigration of spiders (Araneae) into a new polder. Ecological Entomology, 2: $81 \sim 90$

Mochida O and Takada H, 1978. Possible migration of aphid parasites (Hymenoptera: Aphidiidae) across the East China Sea. Applied Entomology and Zoology, 13(2): $125 \sim 127$

Okuma C and Kisimoto R, 1981. Air borne spiders collected over the East China Sea. Japanese Journal Applied Entomology and Zoology, 25: $296 \sim 298$

Ooi P A C, 1979. Flight activities of brown planthopper, whitebacked planthopper, and their predator Cyrtorhinus lividipennis in Malaysia. International Rice Research Newsletter, $4(6): 12$

Rankin M A and Rankin S, 1980. Some factors affecting presumed migratory flight activity of the convergent ladybeetle, Hippodamia convergens ( Coleoptera: Coccinellidae). Bio- logical Bulletin, 158: $356 \sim 359$

Reynolds D R and Wilson M R, 1989. Aerial samples of microinsects at night over central India. Journal of Plant Protection in the Tropics, 6(2): $89 \sim 101$

Riley J R, Reynolds D R and Farrow R A, 1987. The migration of Nilaparvata lugens ( Stal) (Delphacidae) and other Hemiptera associated with rice during the dry season in the Philippines: a study using radar, visual observations, aerial netting and ground trapping. Bulletin of Entomological Research, 77: $145 \sim 169$

Riley J R, Cheng X N, Zhang X X, Reynolds D R, Xu G M, Smith A D, Cheng J Y, Bao A D and Zhai B P, 1991. The long-distance migration of Nilaparvata lugens (Stal) (Delphacidae) in China: radar observations of mass return flight in autumn. Ecological Entomology, 16: $471 \sim 489$

Riley J R, Reynolds D R, Smith A D, Cheng X N, Zhang X X, Xu G M, Cheng J Y, Bao A D, Zhai B P and Wang H K, 1994. Observations on the autumn migration of Nilaparvata lugens (Homoptera: Delphacidae) and other pests in east central China. Bulletin of Entomological Research, 84: 389 $\sim 402$

Riley J R, Reynolds D R, Mukhopadhyay S, Ghosh M R and Sarkar T K, 1995. Long-distance migration of aphids and other small insects in northeast India. European Journal of Entomology, 92: 639 653

Rothschild M, 1971. A large migration of seven-spotted ladybird (Coccinella septempunctata L.) at Deauville, France. Entomologist London, 104: $45 \sim 46$

Rubin A, 1981. Phenology and migration of the seven-spotted ladybird (Coccinella septempunctata L.) on Mount Meron, Israel. Israel Journal of Zoology, 30: 95

Salmon T and Horner N V, 1977. Aerial dispersion of spiders in North Central Texas. Journal of Arachnology, 5: $153 \sim 157$

Way M J and Heong K L, 1994. The role of biodiversity in the dynamics and management of insect pests of tropical irrigated rice-a review. Bulletin of Entomological Research, 84: $567 \sim 587$

Woiwod L P and Harrington R, 1994. Flying in the face of change: the Rothamsted insect survey. In: Leigh $\mathrm{R}$ A, Johnston A E (eds.), Long-term Experiments in Agricultural and Ecological Sciences. UK: CAB International, 321 342

Woiwod L P and Thomas J A, 1993. The ecology of butterflies and moths at the landscape scale. In: Haines-Young $\mathrm{R}$ (ed.), Landscape Ecology in Britain. IALE: Department of Geogvaphy. University of Nottingham, Working paper No. 21: $76 \sim 92$

Woiwod L P and Dancy K J, 1987. Synoptic monitoring for migrant insect in Great Britain and Western Europe. VII. Annual population fluctuations of macrolepidoptera over Great Britain for 17 years. Rothamsted Report for 1986, Part 2: $237 \sim 264$

(责任编辑：时意专) 